\title{
Validation of numerical methods for electromagnetic dosimetry through near-field measurements
}

\author{
D. Giordano ${ }^{1}$, L. Zilberti ${ }^{1}$, M. Borsero ${ }^{1}$, R. Forastiere ${ }^{2}$, W. Wang $^{1}$ \\ ${ }^{1}$ INRIM, strada delle Cacce 91 - 10135 Torino, Italy \\ 2 Dip. Energia, Politecnico di Torino, corso Duca degli Abruzzi - 10129 Torino, Italy
}

\begin{abstract}
This paper describes the arrangement of a first experimental set-up which allows the comparison between the measurement of the electromagnetic field quantities induced inside a simple cylindrical phantom and the same quantities estimated numerically through a boundary element method. The reliability of the numerical method has been tested at $64 \mathrm{MHz}$, the Larmor frequency associated to the magnetic resonance imaging devices with an isocenter magnetic field of $1.5 \mathrm{~T}$. To assess its robustness, the comparison is also performed by introducing, inside the phantom, a metallic non magnetic element, which roughly simulates a medical implant.
\end{abstract}

\section{Section: RESEARCH PAPER}

Keywords: electromagnetic dosimetry; MRI; near-field measurements

Citation: D. Giordano, L. Zilberti, M. Borsero, R. Forastiere, W. Wang, Validation of numerical methods for electromagnetic dosimetry through near-field measurements, Acta IMEKO, vol. 4, no. 1, article 14, February 2015, identifier: IMEKO-ACTA-04 (2015)-01-14

Editor: Paolo Carbone, University of Perugia

Received December $31^{\text {st }}, 2013$; In final form November $21^{\text {st }}, 2014$; Published February 2015

Copyright: (C 2014 IMEKO. This is an open-access article distributed under the terms of the Creative Commons Attribution 3.0 License, which permits unrestricted use, distribution, and reproduction in any medium, provided the original author and source are credited

Funding: European Metrology Research Programme (EMRP)-HLT06 Joint Research Project (JRP) “Metrology for next-generation safety standards and equipment in MRI" (2012-2015)

Corresponding author: D. Giordano, e-mail: d.giordano@inrim.it

\section{INTRODUCTION}

An estimated $8-10 \%$ of the European population are carrying medical implants. At present, they are excluded from receiving a Magnetic Resonance Imaging (MRI) scan as no metrics exists to assess the specific safety risks related to these implants.

With the aim of investigating implant associated risks [13], advanced modelling concepts for the evaluation of electromagnetic field inside phantoms and human computer models [4][5] are fundamental tools which must be validated by experimental comparisons to guarantee their reliability. To this end an experimental tool was designed and set up and first comparisons between measurements and numerical results obtained through a Boundary Element Method (BEM) algorithm, are presented in the paper.

Since this preliminary investigation is based on a simple numerical model involving all the physical aspects, a simplified experimental tool is required as well. To do that a system made of a cylindrical phantom with a tissue-like liquid, a loop antenna and a 3D electromagnetic field mapping was set up.

The magnetic source and measuring system shall be able to generate and detect Radio Frequency (RF) electromagnetic fields with magnitude and frequency similar to the ones exploited by Magnetic Resonance Imaging (MRI) devices for diagnostic purposes. As regards the frequency, it shall be comparable with the isocentre resonance frequency (Larmor frequency) of the most used MRI devices. These are characterized by frequencies of about $64 \mathrm{MHz}, 128 \mathrm{MHz}$ and $300 \mathrm{MHz}$ related to isocentre static magnetic fields of $1.5 \mathrm{~T}, 3 \mathrm{~T}$ and $7 \mathrm{~T}$ respectively. For these preliminary investigations the frequency of interest was set to $64 \mathrm{MHz}$ [6].

As well known from the Nuclear Magnetic Resonance (NMR) theory, a rotation of the nuclear magnetic moment vectors can be impressed by transferring a suitable amount of energy into the anatomy by means of coils tuned at the Larmor frequency. Such coils have to generate RF magnetic 


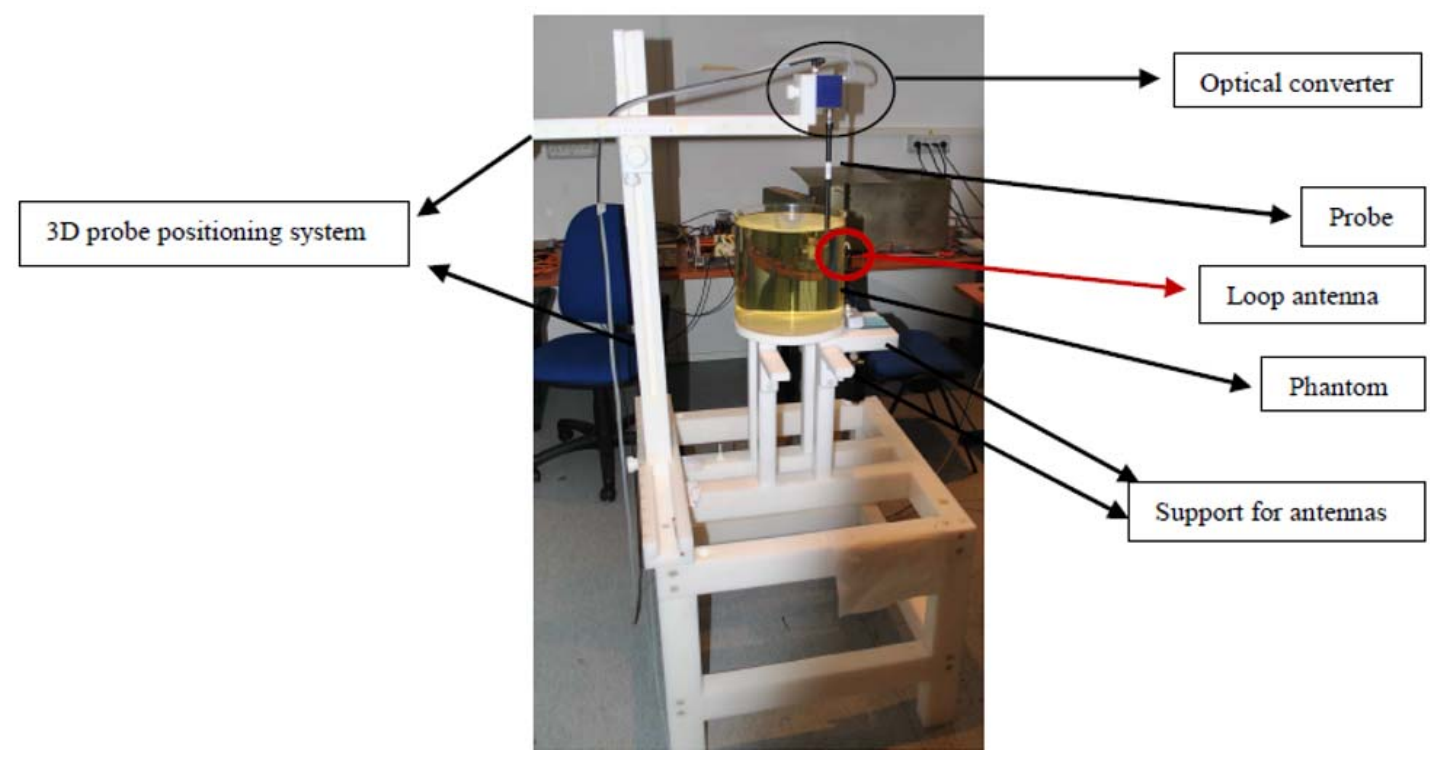

Figure 1 Experimental set-up.

fields perpendicular to the static magnetic field direction. Consequently, a loop antenna was selected [6-7].

Since the aim of this paper is the experimental set-up, just a synthetic description of the field formulation is given in section 2 .

A description of the experimental set-up is given in section 3, and some critical items due to the simplified model of the loop antenna are pointed out. In particular, the presence of the shield in the actual loop and the absence of the earth in the numerical model are argued.

The first comparisons between measured and computed magnetic field components are performed along a vertical and a radial line inside the cylindrical phantom. Additional comparisons are performed when a metallic object mimicking an implant is introduced inside the phantom (see section 4).

A brief description of the system control and operations, managed by a Python programme which automates the generation and acquisition of the field components, is given in section 5 , followed by some conclusions (section 6 ).

\section{FIELD FORMULATION}

The electromagnetic field problem is described by the Electric Field Integral Equation (EFIE) and Magnetic Field Integral Equation (MFIE) under sinusoidal conditions (angular frequency $\omega$ ). The integral equations are solved by the Boundary Element Method (BEM), discretizing the external surface $(\partial \Omega)$ of the considered objects $(\Omega)$ with $2 \mathrm{D}$ surface elements. The discretized form of the EFIE and MFIE equations is:

$$
\begin{aligned}
\xi \boldsymbol{E}_{i}= & -\int_{\Omega_{s}} j \omega \mu \Psi \boldsymbol{J}_{s} d v-\sum_{m}^{M}(\boldsymbol{n} \times \boldsymbol{E})_{m} \times \int_{\partial \Omega_{m}} \nabla \Psi_{i, m} d s \\
& -\sum_{m}^{M}(\boldsymbol{n} \cdot \boldsymbol{E})_{m} \int_{\partial \Omega_{m}} \nabla \Psi_{i, m} d s+j \omega \mu \sum_{m}^{M}(\boldsymbol{n} \times \boldsymbol{H})_{m} \int_{\partial \Omega_{m}} \Psi_{i, m} d s
\end{aligned}
$$

$$
\begin{aligned}
\xi \boldsymbol{H}_{i} & =\int_{\Omega_{s}}\left(\boldsymbol{J}_{s} \times \nabla \Psi\right) d v-\sum_{m}^{M}(\boldsymbol{n} \times \boldsymbol{H})_{m} \times \int_{\partial \Omega_{m}} \nabla \Psi_{i, m} d s \\
& -\sum_{m}^{M}(\boldsymbol{n} \cdot \boldsymbol{H})_{m} \int_{\partial \Omega_{m}} \nabla \Psi_{i, m} d s-j \omega \tilde{\varepsilon} \sum_{m}^{M}(\boldsymbol{n} \times \boldsymbol{E})_{m} \int_{\partial \Omega_{m}} \Psi_{i, m} d s
\end{aligned}
$$

where $J_{\mathrm{s}}$ is the impressed current density of the sources $\left(\Omega_{\mathrm{s}}\right)$, $\xi$ is the singularity factor $(\xi=0.5$ on the surface and $\xi=1$ elsewhere) and $n$ is the normal unit vector directed outwards $\Omega$. The $\mathrm{m}$-th element is the source point while, during the setting of the matrix, the computational point is the barycentre of the i-th element. The Green function is defined as:

$\psi=\frac{e^{-i k\left|\boldsymbol{r}-\boldsymbol{r}^{\prime}\right|}}{4 \pi\left|\boldsymbol{r}-\boldsymbol{r}^{\prime}\right|}$ with $k=\omega \sqrt{\mu\left(\varepsilon-j \frac{\sigma}{\omega}\right)}=\omega \sqrt{\mu \tilde{\varepsilon}}$

where $r$ and $r$ ' are the coordinate vectors of the observation and of the source points, while $\mu, \sigma$ and $\varepsilon$ are the magnetic permeability, the electric conductivity and the electric permittivity respectively. EFIE and MFIE can be written for any volume belonging to the computational domain (the air region outside the phantom, inside this latter and also inside possible metallic objects). The volume integrals must be included only in volumes involving impressed sources.

The integrals of the Green function and its gradient are computed by using an adaptive quadrature rule based on Kronrod algorithm, which enables high accuracy limiting the computational burden.

\section{EXPERIMENTAL SET-UP}

Figure 1 shows an overall view of the experimental setup, including the phantom filled with a yellow liquid (the human tissue-like liquid), the $3 \mathrm{D}$ probe positioning system made of dielectric material with low permittivity $\left(\varepsilon_{\mathrm{r}}=2\right)$, the electric (or magnetic) field probe and its optical converter, the support for the antennas (more antennas are foreseen). The black loop antenna can be seen on the right side of the phantom. 

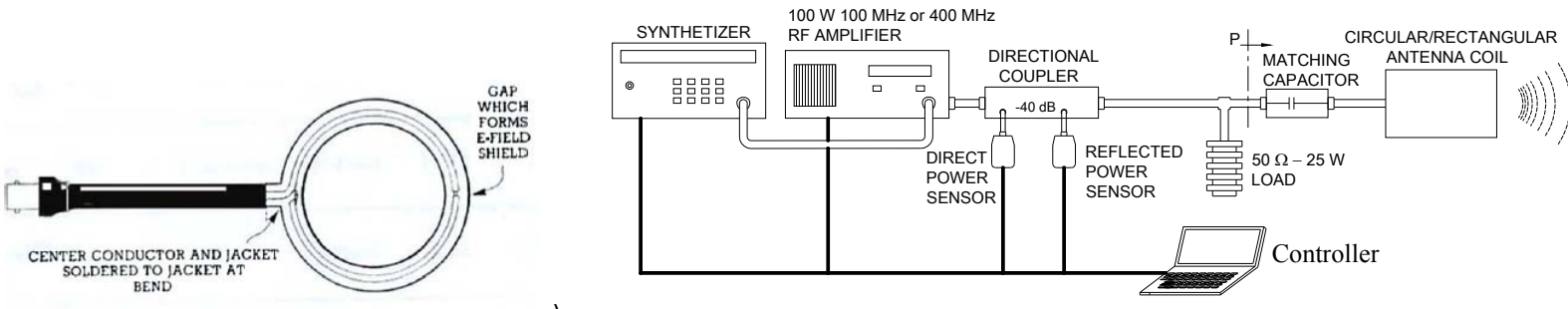

a)

Figure 2. a) Sketch of the loop antenna [8]. b) Sketch of the set-up for the generation of RF magnetic field.

\subsection{Phantom}

The phantom consists of a cylindrical container made of polymethylmethacrylate whose dielectric permittivity $\left(\varepsilon_{\mathrm{r}}=\right.$ 2.2), much lower than that of the liquid, does not affect the electric field induced in the phantom. It has both an internal diameter and an internal height equal to $240 \mathrm{~mm}$. The liquid, prepared by the Physikalisch Technische Bundesanstalt laboratories (PTB, Berlin), shows electrical characteristics comparable with the ones of some human tissues.

To reduce the effects of the support material on the electric field behaviour around the phantom, four slim columns sustain the container, increasing the distance from the structure supporting the whole system.

\subsection{Magnetic field generation system}

The magnetic field source shall be able to induce, inside the phantom, an electric field with a magnitude detectable by the near-field probes employed for this experiment. Moreover, the source should not produce spurious electric
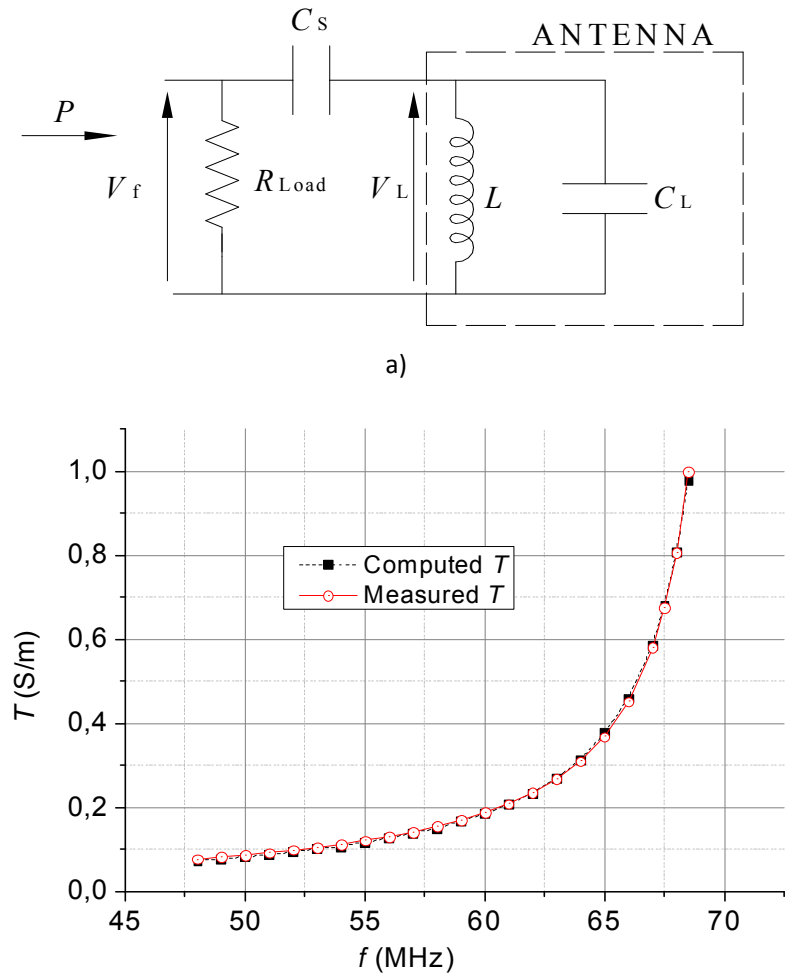

b)

Figure 3. a) Circuital model for the estimation of the current flowing in the loop antenna. b) Computed and measured frequency behaviour of $T$ coefficient. fields which are not considered in the numerical procedure.

The selected source for these purposes is a small loop antenna, consisting of a single turn with $6 \mathrm{~cm}$ diameter, inside a balanced E-field shield (Figure 2a).

Through preliminary computations, a magnetic field amplitude of $1 \mathrm{~A} / \mathrm{m}$ produced by a current of $0.5 \mathrm{~A}$ is predicted at $20 \mathrm{~mm}$ far from the loop. Of course this value is lower than the typical magnetic field value generated by the RF imaging coil of the MRI devices [9-11]; nevertheless, this is acceptable because the purpose here is not to cause the nuclear magnetic resonance but to investigate the possible side-effects induced in the human body during MRI examination. The estimated electric field induced by such magnetic field is a few tens of volts per meter, enough to be detected by the probe.

A first experimental arrangement for the supply and estimation of the current flowing in the antenna is depicted in Figure 2b). The supply system consists of a signal generator and a $100 \mathrm{~W}$ broadband amplifier $(9 \mathrm{kHz}$ to 100 $\mathrm{MHz}$ ). In order to improve the matching between the supply system and the antenna, a series capacitor $C s$, with a rated value of $8 \mathrm{pF}$, is introduced. In this way it is possible to obtain the required field without exceeding the maximum power $(25 \mathrm{~W})$ of the $50 \Omega$ resistive load. This load allows to estimate the current which generates the field. Indeed, through the knowledge of the voltage across the resistive load, obtained by the measurement of the incident power $P$, it is possible to solve the circuital model shown in Figure $3 a$ and to estimate the current that flows in the inductance $L$ simulating the loop antenna. The parameter $C_{L}$ simulates the stray electrical coupling between the loop wire and its shield. Because of the antenna dimensions and supply frequency, the radiation resistance can be neglected as well as the Joule losses and the circuital model can be simplified.

The current flowing in the loop antenna (and hence in the inductance $L$ ), $\mathrm{L}$, which generates the magnetic field,

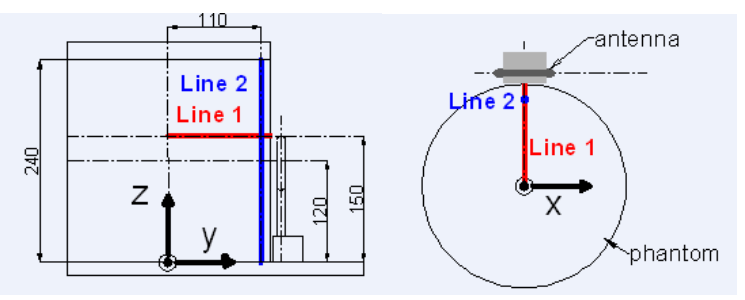

Figure 4. Investigation lines inside the phantom: frontal and upper view. 
can be directly estimated by knowing the transmitted power to $R_{\text {Load }}$ and the admittance $T$ defined as:

$|\bar{T}|=\frac{\left|\bar{I}_{L}\right|}{\left|\bar{V}_{f}\right|}=\frac{\omega \cdot C_{s}}{1-\left(\frac{\omega}{\omega_{\text {res }}}\right)^{2}} \quad$ with $\quad \omega_{\text {res }}=\frac{1}{\sqrt{L \cdot\left(C_{L}+C_{s}\right)}}$

The transmitted power is obtained as difference between the incident and reflected power values measured by means of a directional coupler and a power meter. From that, the voltage $V_{\mathrm{f}}$ is easily obtained.

The unknown $C_{s}$ and $\omega_{\text {res }}$ are extrapolated from the experimental frequency behaviour of $T$ from $45 \mathrm{MHz}$ to 70 $\mathrm{MHz}$ by means of a least square algorithm between the measured frequency behaviour and the computed one.

Assuming that the current is uniform along the single turn (i.e., $\pi D<<\lambda / 10$, where $D$ is the turn diameter and $\lambda$ is the wavelength of the working frequency), the magnetic field in the loop centre is $H_{c}=\frac{I_{L}}{D}$; then the following system can be written:

$$
\left\{\begin{array}{l}
|\bar{T}|\left(\omega_{1}\right)=\left.D \frac{H_{c}}{\left|\bar{V}_{f}\right|}\right|_{\omega_{1}}=\frac{\omega_{1} \cdot C_{s}}{1-\left(\frac{\omega_{1}}{\omega_{\text {res }}}\right)^{2}} \\
|\bar{T}|\left(\omega_{2}\right)=\left.D \frac{H_{c}}{\left|\bar{V}_{f}\right|}\right|_{\omega_{2}}=\frac{\omega_{2} \cdot C_{s}}{1-\left(\frac{\omega_{2}}{\omega_{r e s}}\right)^{2}}
\end{array}\right.
$$

where $\omega_{1}$ and $\omega_{2}$ are arbitrary angular frequencies chosen in the range $45 \mathrm{MHz}$ to $70 \mathrm{MHz}$. From a series of measured values $\frac{H_{c}}{V_{f}}\left(\omega_{i}\right)$ evaluated in the same frequency range a couple of such values is randomly extracted to solve the system (4) obtaining a series of couple of values (Cs-i, $\omega_{\text {res }}$ i). By means of the above cited least square algorithm between the measured $T(\omega)$ values and the computed ones, the optimal couple of values $\left(C s, \omega_{\text {res }}\right)$ were found to be equal to $8.3 \mathrm{pF}$ and $70.6 \mathrm{MHz}$, respectively. Figure $3 \mathrm{~b}$ shows the frequency behaviour of the computed and measured $T$ coefficient.

The gap on the E-field shield such as a coupling between the antenna and earth, due to a non-symmetric supply, can compromise the comparison between measurement and numerical results. Indeed the two elements introduce spurious electric components which are not taken into account by the numerical procedure. A non-symmetric supply for the antenna generates a common electric coupling with the earth evidenced by an electric field component perpendicular to the earth, not foreseen by the model. The gap problem can be overcome by shielding the gap with a metallic strip. The earth coupling disappears when the antenna is placed close to the phantom. The conductivity of the liquid "shields" the antenna from the earth. The encouraging results shown in the following prove the reliability of the assertions.

\subsection{Electric and magnetic field probes}

Since the electromagnetic field measurement should be performed in the near-field region, probes with very small dimensions are chosen. Moreover, owing to their usage inside the phantom, resistance to organic solvent must be guaranteed. Their main characteristics are resumed in Table I [12].

These isotropic probes give the true rms value of the applied field along the three orthogonal axes. Thanks to an electro-optic converter the information can be easily carried from the probe to the meter which can be placed a few meters away from the magnetic source.

The field generation and detection system is automatically managed by a Python program which reduces the measurement time and increases the accuracy of the power measurement, allowing for a thermal equilibrium of the devices.

\section{COMPARISON BETWEEN NUMERICAL MODEL AND MEASUREMENT RESULTS}

A preliminary calibration phase gives evidence of a rotation of the $x / y$ field arrays around the $z$ direction with respect to the absolute reference system shown in Figure 5, for both the electric and magnetic field probes. The estimation of such rotation angle is performed thanks to the symmetry conditions imposed by the loop antenna. The results, accompanied by an uncertainty of about $10 \%$, are $20^{\circ}$ and $23.5^{\circ}$ for the electric and magnetic probe respectively. The algorithm which manipulates the acquired field components to apply the coordinate rotation is implemented in the software which controls the whole experimental set-up (section 5).

As a first comparison between the BEM algorithm and

Table 1. Characteristics of the electric and magnetic field probes.

\begin{tabular}{lcc}
\hline \multicolumn{1}{c}{ Section } & Electric field & Magnetic Fied \\
\hline Frequency & $40 \mathrm{MHz} \div 6 \mathrm{GHz}$ & $10 \mathrm{MHz} \div 600 \mathrm{MHz}($ absolute accuracy $\pm 6.0 \%$ ); \\
output linearized & $0.08 \mathrm{~A} / \mathrm{m} \mathrm{to} 40 \mathrm{~A} / \mathrm{m} \mathrm{at} 13.56 \mathrm{MHz}$ \\
Dynamic range & $2 \mathrm{~V} / \mathrm{m} \div 1000 \mathrm{~V} / \mathrm{m}$ & $0.01 \mathrm{~A} / \mathrm{m}$ to $5 \mathrm{~A} / \mathrm{m}$ at $100 \mathrm{MHz}$ \\
& $337 \mathrm{~mm}$ (tip: $40 \mathrm{~mm})$ & $337 \mathrm{~mm}$ (tip: $40 \mathrm{~mm})$ \\
$\begin{array}{l}\text { Overall length } \\
\begin{array}{l}\text { Distance from } \\
\text { probe tip to dipole centre }\end{array}\end{array}$ & $2.5 \mathrm{~mm}$ & $3 \mathrm{~mm}$ \\
Tip diameter & $8 \mathrm{~mm}$ (body: $10 \mathrm{~mm})$ & $6 \mathrm{~mm}$ (body: $12 \mathrm{~mm})$ \\
\hline
\end{tabular}




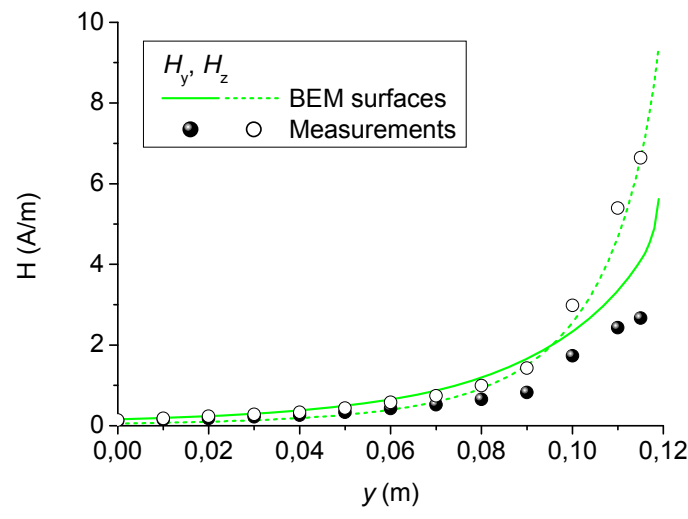

a)

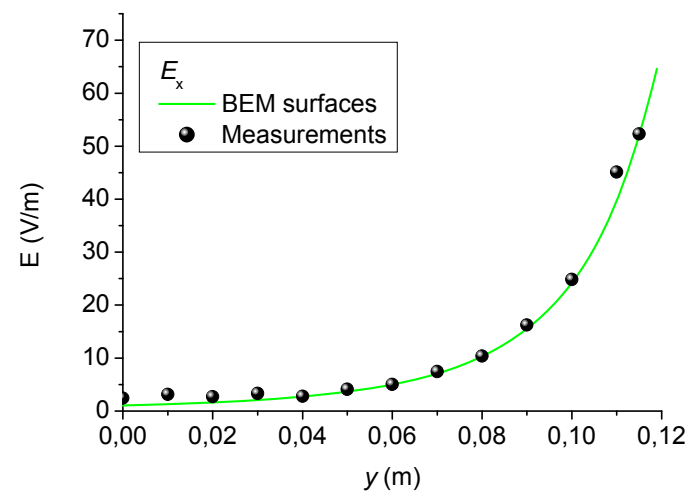

b)

Figure 5. Magnetic a) and electric b) r.m.s. field behaviour along line 1 of the three components with a unit-current in the antenna.

measurements, two investigation lines are chosen. Line 1 is in parallel to the $y$ axis (Figure 4), $30 \mathrm{~mm}$ above it. Since the induced current effects on the magnetic field at $64 \mathrm{MHz}$ are negligible, the ideal magnetic field array lies on $y$-z plane, so the $H_{x_{-} \text {BEM }}$ component is null (Figure $5 \mathrm{a}$ ). The electric field lines induced in the phantom are, at this frequency, circular with the centres on the loop axis, thus, only $E_{\mathrm{x}_{-} \mathrm{BEM}}$ is non-zero along the investigation line (Figure $5 \mathrm{~b})$.

Line 2 is in parallel to the $z$ axis and is placed $110 \mathrm{~mm}$ from the reference system centre. Since this line lies on the $z-y$ plane which cuts symmetrically the antenna, the $H_{\mathrm{x}_{-} \mathrm{BEM}}$

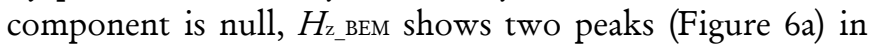
correspondence with the minimum distance between line 2 and the turn of the antenna, while $H_{\mathrm{y}_{-} \text {BEM reaches the peak }}$

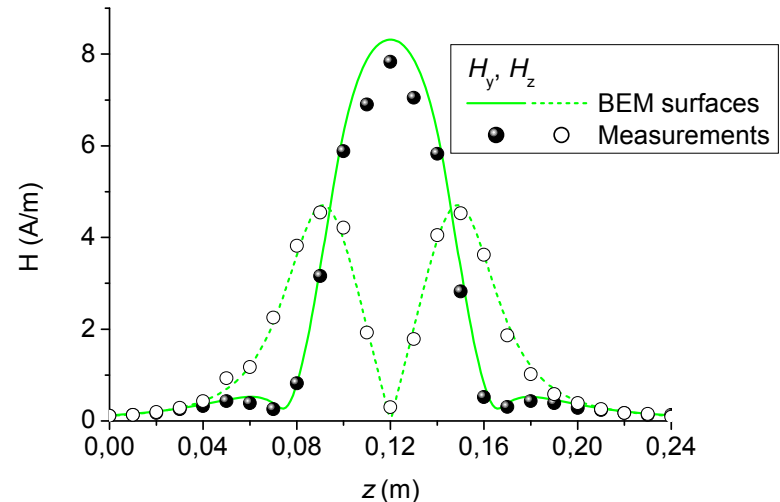

a)

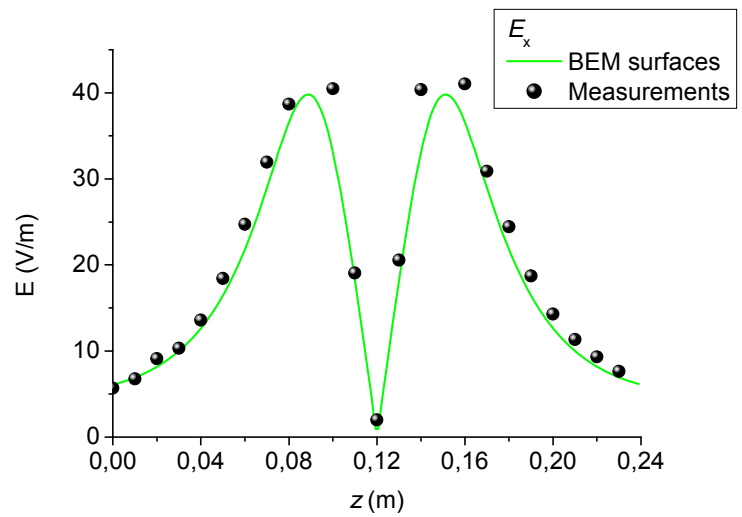

b)

Figure 6. Magnetic a) and electric b) r.m.s. field behaviour along line 2 of the three components with a unit-current in the antenna.

in correspondence with the loop antenna axis. The shape of the $E_{\mathrm{x}_{-} \mathrm{BEM}}$ component should be analogous to the shape of $H_{z_{-} \text {BEM }}$ while $E_{\mathrm{y}_{-} \mathrm{BEM}}$ and $E_{\mathrm{z}_{-} \mathrm{BEM}}$ are zero (Figure $6 \mathrm{~b}$ ) because of the circular shape of the electric field lines.

When performing electric field measurements the noise level is not always negligible (in the order of 2 to $3 \mathrm{~V} / \mathrm{m}$ ). To reduce the noise effects on the measured values, the following expression is employed:

$E_{64 M H z}=\sqrt{E_{\text {tot }}^{2}-E_{\text {noise }}^{2}}$

where $E_{64 \mathrm{MHz}}$ is the electric field corrected for the noise, $E_{\text {tot }}$ is the electric field given by the meter (signal plus noise) and $E_{\text {noise }}$ is the mean value of the noise detected when the loop antenna is not supplied.

In spite of the problems spotted both in the estimation of the current flowing in the antenna at $64 \mathrm{MHz}$ and the
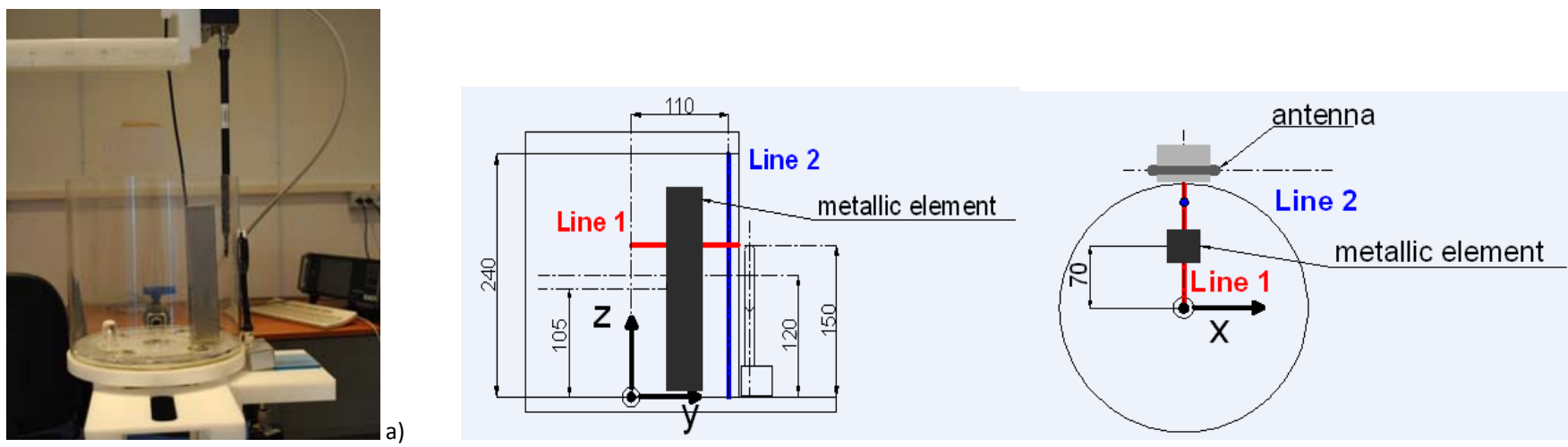

b)

Figure 7 a). Metallic parallelepiped $200 \mathrm{~mm}$ high placed inside the phantom. b) investigation lines and dimensions : frontal and upper view. 


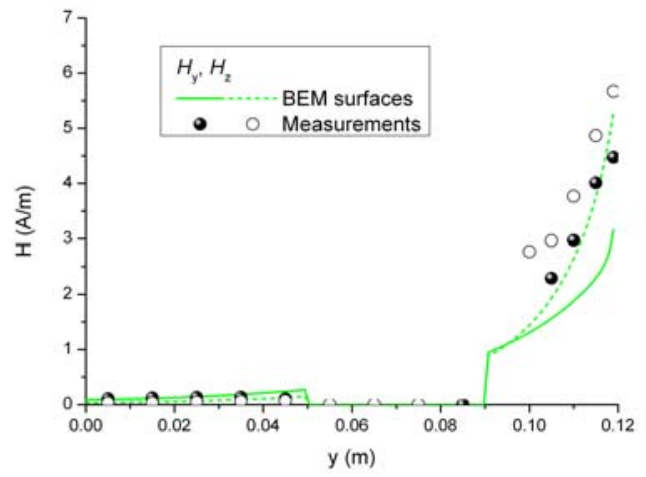

a)

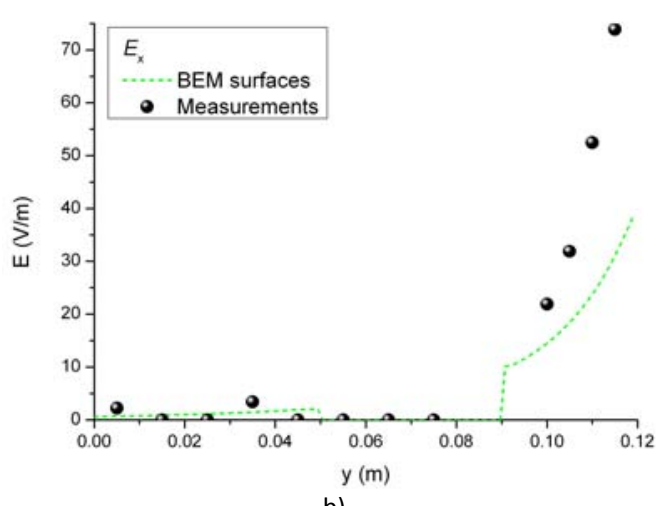

b)

Figure 8 Magnetic a) and electric b) r.m.s. field behaviour along line 1 of the three components with a unit-current in the antenna.

approximation introduced in the numerical representation of the antenna, the measurement-computation comparisons give encouraging results. In particular, Figures $6 a$ and $6 b$ show the very good performance of the probes which can detect field having high spatial gradient (close to the antenna axis: $z=0.12 \mathrm{~m}$ ).

The experimental scenario is finally made more complicated by introducing a $40 \times 40 \times 240 \mathrm{~mm}^{3}$, conductive, non-magnetic parallelepiped. The aim is to roughly simulate a medical implant. Its large dimensions provide a high distortion field, allowing a first evaluation of the sensitivity of the numerical method. Figure 7 shows a picture of the metallic object inserted in the phantom and its position.

Figures 8 and 9 provide the numerical results compared with the measured ones for the same lines previously investigated. As can be easily seen, a higher discrepancy between measurement and computation is detected, in particular for line 2. This fact deserves further investigations, but probably it can be ascribed to the features of the problem matrix, which, in presence of the metallic object, is badly scaled and gives rise to a nonoptimal convergence of the iterative solver used to solve the BEM formulation. Moreover, the wavelength in the metallic object is much lower than elsewhere and this would ask for a very fine mesh, with a strong increase of the computational burden needed to guarantee an accurate reconstruction of the electromagnetic quantities.

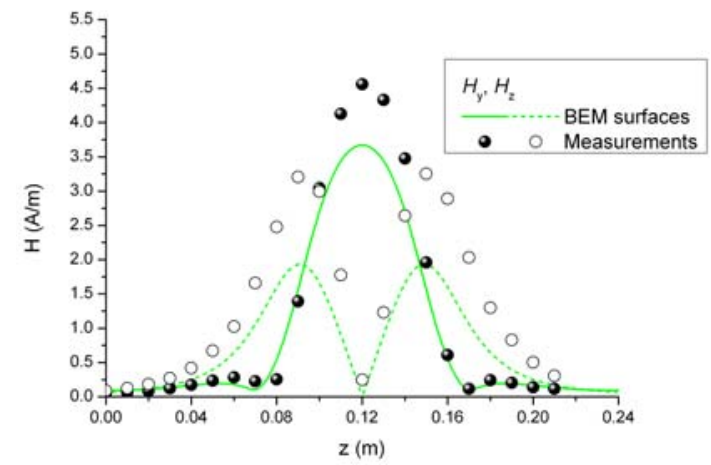

a)

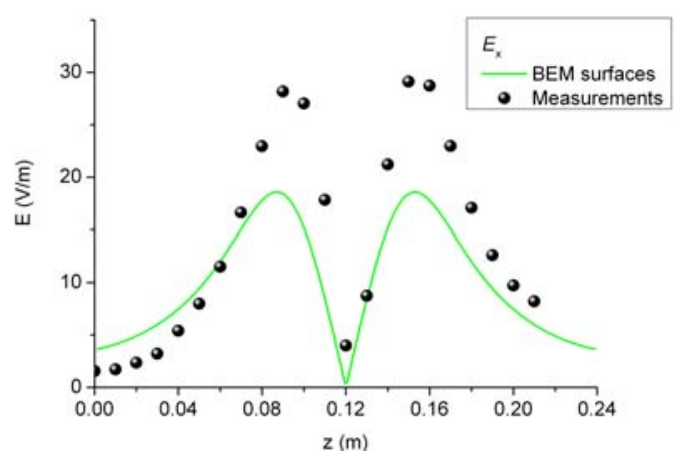

b)

Figure 9. Magnetic a) and electric b) r.m.s. field behaviour along line 2 of the three components with a unit-current in the antenna.

\section{SYSTEM CONTROL AND OPERATIONS}

In order to increase the safety, measurement reliability and speed, the supply and measurement systems are managed by a software developed in Python environment.

Python is an interpreted, general purpose, high-level programming language [13].

The software controls the synthesizer and power amplifier by means of a GPIB-USB controller. The acquisition of the incident and reflected power is supervised by an USB connection. To download the acquired electric/magnetic field components, a simple network between the remote unit EASY4 (server) and the PC (client) by an ETHERNET connection [14] is set.

Thanks to a console user interface it is possible to insert the working directory and the output file names. If the directory and/or the files do not exist the program creates them.

The measurement results are stored in two output files with the same name but different extensions: “.txt". They contain the per-unit current electric/magnetic field components, referred to the main reference coordinate, while the second file, with a ".raw" extension, contains the raw field components, affected by noise, and referred to a rotated coordinate system. Moreover, the simple user interface requires: the type of the employed probe (electric or magnetic field probe), the geometrical characteristics of the investigation curve and the number of the measurement points. 
Once the required measurement attributes are inserted and the electric field probe is connected, the software makes the noise level detection available for each component. Then, a warm-up period of $60 \mathrm{~s}$ is run to guarantee a thermal equilibrium of the whole system; after that the remote unit provides the field components which are downloaded. In the same time the power information is downloaded and, thanks to the circuital model of the antenna, the flowing current is estimated. A suitable module of the software provides the rotation of the field array in order to refer to the main coordinate system, cancels the noise and evaluates the per-unit current field components.

After this phase, the output software gives the coordinate of the new investigation point, the operator places the probe in the indicated point and by pushing enter a new supply, acquisition and manipulation phase is run.

In order to protect the matching capacitors, a control structure gives a warning signal if the voltage across the capacitors, estimated on the base of the antenna circuital model and of the voltage and frequency values set on the synthesizer, exceeds a defined limit.

\section{CONCLUSIONS}

A description of a first experimental set-up devoted to the assessment of the reliability of numerical models for the estimation of induced electromagnetic fields into a phantom simulating human tissues is presented. A simple model of the transmitting antenna with lumped parameters is implemented and an evidence of the effectiveness of the model is given up to about $70 \mathrm{MHz}$.

A first comparison between computation and measurement along two investigation lines inside the phantom is shown. The results are encouraging. The computed electric field over line 1 shows a maximum deviation lower than $8 \%$ for field magnitudes higher than 5 $\mathrm{V} / \mathrm{m}$. For line 2, characterized by a higher field gradient value, the computed electric field has a maximum deviation lower than $20 \%$ for field magnitudes higher than $5 \mathrm{~V} / \mathrm{m}$. The magnetic field comparison between computation and measurement over line 1 shows a deviation higher than $30 \%$ for the $H_{\mathrm{y}}$ component, while the $H_{\mathrm{z}}$ component is better reproduced. The estimated magnetic field over line 2 reproduces with high fidelity the measured one, except for the peak value of the $H_{y}$ component, for which a deviation of $6 \%$ is detected.

To deeper investigate the reliability of the numerical model in presence of medical implants, the measurements are carried out by placing a metallic object within the tissue-simulating liquid in the phantom. Even though the comparison between measurement and numerical method is acceptable, a higher deviation is found, in particular for the peak values of each component. Indeed, a metallic object with high conductivity introduces a spatial discontinuity of this parameter and can reduce the accuracy of the numerical simulation.

Presently, the design of a new antenna system is being developed to increase the value of the electric and magnetic field-strength generated in the phantom, and a further measurement campaign is in progress.

\section{ACKNOWLEDGEMENT}

The authors wish to thank Dr. Gerd Weidemann from PTB for his contribution in the production and characterization of the human tissue-like liquid.

\section{REFERENCES}

[1] IEC 62311 "Assessment of electronic and electric equipment related to human exposure restrictions for electromagnetic fields ( $0 \mathrm{~Hz}-300 \mathrm{GHz}), 2007$.

[2] Koch, K.M. and Hargreaves, B.A. and Pauly, K. Butts and Chen, W. and Gold, G.E. and King, K.F, "Magnetic resonance imaging near metal implants", Journal of Magnetic Resonance Imaging, vol.32, no.4, pp.773-787, 2010.

[3] Ho, Henry S., "Safety of metallic implants in magnetic resonance imaging", Journal of Magnetic Resonance Imaging, vol.14, no.4, pp.472-477, 2001.

[4] M. Borsero, O. Bottauscio, L. Zilberti, M. Chiampi, W. Wang "A Boundary Element estimate of radiated emissions produced by unknown sources", Electromagnetic Compatibility (EMC EUROPE), 2012 International Symposium on.

[5] Oriano Bottauscio, Mario Chiampi, Luca Zilberti “Boundary element approach to relate surface fields with the specific absorption rate (SAR) induced in 3-D human phantoms", Eng. Analysis with Boundary Elements vol 35 (2011) pp. 657-666.

[6] "RF coils for MRI", J. ThomasVaughon, John r. Griffiths, John Villy \& Sons Ltd, 2012.

[7] Joseph P. Hornak "The Basics of MRI", www.cis.rit.edu/htbooks/mri/, 1996.

[8] "Near Field Probe Set User's Manual”, EMCO The electro Mechanics Company, 1988.

[9] Stefano Pisa, et all. "A study of the Interaction Between Implanted Pacemakers and the Radio-Frequency field Produced by Magnetic Resonance Imaging apparatus", IEEE trans. on EMC, vol. 50, NO.1, pp. 35-42, February 2008.

[10] Tsuboi, H.; Tanaka, H.; Misaka, H.; Fujita, M., "Electromagnetic field analysis of RF antenna for MRI," Magnetics, IEEE Transactions on, vol.24, no.6, pp.2591,2593, Nov 1988.

[11] Fujita, M. and Higuchi, M. and Tsuboi, H. and Tanaka, H. and Misaki, T., "Design of the RF antenna for MRI", Magnetics, IEEE Transactions on, vol.26, no.2, pp.901-904, 1990.

[12] SPEAG, "EASY4/MRI" www.speag.com/products/easy4$\mathrm{mri} /$ probes-2.

[13] "Introduction to Python Programming and developing GUI Applications with PyQT", B.M. Harwani, Course technology a part of cengage Learning, 2012.

[14] EASY4 Exposure Acquisition System, Schmid \& Partner Engineering AG February 3, 2010. 